九州大学学術情報リポジトリ

Kyushu University Institutional Repository

\title{
Mathematieal Modeling of Fish Schooling of Japanese Medaka using Basic Behavioral Patterns
}

Hiramatsu, Kazuaki

Laboratory of Drainage and Water Environment, Division of Regional Environment Science, Department of Bioproduction Environmental Science, Faculty of Agriculture, Kyushu University

Shikasho, Shiomi

Laboratory of Drainage and Water Environment, Division of Regional Environment Science, Department of Bioproduction Environmental Science, Faculty of Agriculture, Kyushu University

Mori, Ken

Laboratory of Bioproduction and Environment Information Sciences, Division of Bioproduction and Environment Information Sciences, Department of Bioproduction Environmental Science, Faculty of Agriculture, Kyushu University

https://doi.org/10.5109/24374

出版情報: 九州大学大学院農学研究院紀要. 45 (1)，pp.237-253，2000-11. Kyushu University バージョン：

権利関係 : 


\title{
Mathematical Modeling of Fish Schooling of Japanese Medaka using Basic Behavioral Patterns
}

\author{
Kazuaki Hiramatsu, Shiomi Shikasho and Ken Mori* \\ Laboratory of Drainage and Water Environment, Division of Regional Environment Science, \\ Department of Bioproduction Environmental Science, Faculty of Agriculture, \\ Kyushu University, Fukuoka 812 8581, Japan \\ (Received June 27, 2000 and accepted August 18, 2000)
}

\begin{abstract}
A mathematical model was proposed to describe the behavior of fish schooling in a water tank without the presence of flow. First, video images of schooling Japanese Medaka fish (Japanese killifish, Oryzias latipes) in the water tank were analyzed to determine the interactions between individuals: the spacing between individuals, the spatial distribution of individuals and the communication processes. The behavioral pattern model was then applied to the investigation to simulate the schooling phenomenon. In the behavioral pattern model, the behavior of a single fish was based on four patterns: the repulsion, the parallel orientation, the attraction and the searching. The influence of neighbors was averaged by an arithmetic mean. The optimal model structure was searched by a simple genetic algorithm using the experimental data. The results of the simulations agreed with the experimental data in many regards, including the polarization, the nearest neighbor distance, the expanse and the fractal dimension.
\end{abstract}

\section{INTRODUCTION}

Fish behavior is one of the essential indicators, not only for evaluating the water environment following the impact of development activities on river ecosystems, but also for evaluating the functional aspects of river improvement. In contrast to the past ecological studies that have tried to explain the preferences of fish from field measurements in a natural habitat, our study is directed toward developing a methodology for such evaluations with the aid of numerical simulation techniques. The final goal of this study is to evaluate the fish preference in a hydraulic environment by numerical simulations with a fish school model and the observed hydraulic data around the hydraulic structures. In this paper, as the first step of this study, a mathematical model will be proposed to describe the behavior of fish schooling in a water tank without the presence of flow.

Nowadays, there may be two fundamentally different approaches to the modeling of a fish school. The first is based on the assumption that the motion of each fish is prescribed by Newton's second law of motion. This approach is called the Newtonian dynamics model. In this approach, the behavior of each fish is expressed by a set of equations of motion which contain external forces representing the interactions with other fish and the effects of environmental fields. The models of Okubo (1986), Sannomiya and Matuda

\footnotetext{
* Laboratory of Bioproduction and Environment Information Sciences, Division of Bioproduction and Environment Information Sciences, Department of Bioproduction Environmental Science, Faculty of Agriculture, Kyushu University
} 
(1984) and Niwa (1994) were based on this approach.

The second one is based on a more biological modeling concept. In this approach, basic behavioral patterns determine how a fish would act if it had only one neighbor fish. The influence of several neighbors is then estimated by a mixture element, such as an arithmetic mean. This approach is called the behavioral pattern model. The models of Aoki (1982), Huth and Wissel (1992) and Reuter and Breckling (1994) were based on this approach.

In this paper, the applicability of the behavioral pattern model to the schooling behavior of Japanese Medaka fish (Japanese killifish, Oryzias latipes) will be tested by numerical simulations against the water tank experiments without the presence of flow. A simple genetic algorithm (SGA) will be incorporated into the modeling to help search for the optimal model structure.

\section{METHOD AND DATA}

\section{Water tank experiment}

Water tank experiments were executed in order to examine the schooling behavior of Japanese Medaka. The Japanese Medaka was once one of the most popular fish which inhabited small streams and paddy fields in the countryside of Japan. However, they have been slowly disappearing in the countryside due to the development activities and they are now listed in the Japanese Red Data Book, published by Environment Agency of Japan, as rare and endangered species. In recent years, the Japanese Medaka is regarded as the symbolic fish of the restoration of countryside ecosystems. In the water tank experiments, we used about 20 Japanese Medaka ranging in body-length from 2.6 to $3.1 \mathrm{~cm}$. The average body-length $d_{B}$ of the experimental fish was $2.8 \mathrm{~cm}$.

In the experiments, we used a colorless and transparent acrylic tank that was $98 \mathrm{~cm}$ square and $10 \mathrm{~cm}$ deep. The water in the tank was kept $5 \mathrm{~cm}$ deep in order to approximate the inside of the tank to be 2-dimensional. The trajectories of the swimming fish were measured in a dark room by means of a digital video camera (Sony Inc., DCR-TRV900) installed at $245 \mathrm{~cm}$ above the water surface in the center of the tank. Fluorescent lamps were utilized as a light-source. Five $40 \mathrm{~W}$ lamps were fixed at $30 \mathrm{~cm}$ below the tank bottom and a white acrylic board with a thickness of $2 \mathrm{~mm}$ was also placed on the tank bottom to uniformly light up the inside of the tank. Black acrylic boards were stuck to the four sides of the walls of the tank to avoid the reflections of the fish and the lamps. In addition, screens were put up around the tank in order not to disturb the fish movement by the figure of an experimenter. Before the experiment, five fishes (the number of individuals $n=5$ ) were transferred from the holding tank to the experimental tank. After the adequate time for accustoming the fish to a new environment, the trajectories of the swimming fish were recorded with the video camera. All experiments were executed at a nearly constant water temperature $\left(20.0-21.5^{\circ} \mathrm{C}\right)$.

The instantaneous images were taken from the recorded video images and then taken onto a personal computer at $1 / 30 \mathrm{~s}\left(=\delta t_{e}\right)$ interval for a $20 \mathrm{~s}$ period per each recording session. Using motion analyzer software (Library Inc., Move- Tr 32/2D), the 2-dimensional coordinates on a horizontal plane were calculated for every fish at $1 / 30 \mathrm{~s}$ interval from the instantaneous images. Final trajectories were obtained through 11-term moving 


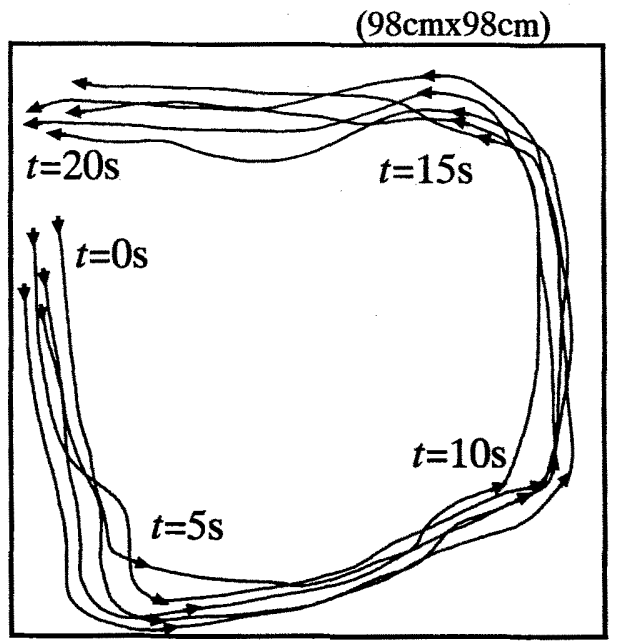

Fig. 1. Moving-averaged trajectories of five Japanese Medaka fish observed in the water tank experiment of Run 1 .

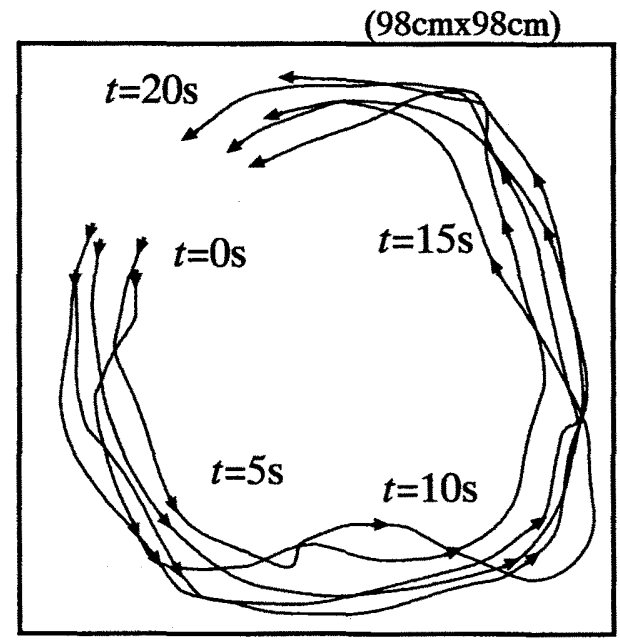

Fig. 2. Moving-averaged trajectories of five Japanese Medaka fish observed in the water tank experiment of Run 2. 
average to eliminate the fluctuations in trajectory caused by the errors occurring in the video image processing. The number of moving average, i.e. 11 terms, was equivalent to $1 / 3 \mathrm{~s}$ which corresponds to the experimental data of Partridge $(1980,1981)$ and Partridge and Pitcher (1980) concerning the reaction time of fish.

In the following section, we will discuss the schooling behavior using two recorded sessions, as shown in Figs. 1 and 2 and Table 1. Figs. 1 and 2 show that the two schools swim counterclockwise along the side walls and also suggest that the school is more cohesive for Run 1 than for Run 2.

\section{Swimming velocity}

The swimming velocity $\left\{v_{i}(t), i=1 \cdots, n\right\}$ for individual $i$ at each time step $t$ were calculated by the position vectors $\left\{X_{i}(t), i=1 \cdots, n\right\}$ :

$$
\begin{aligned}
& V_{i}\left(t+\delta t_{e}\right)=\frac{X_{i}\left(t+\delta t_{e}\right)-X_{i}(t)}{\delta t_{e}}, \\
& v_{i}\left(t+\delta t_{e}\right)=\left\|V_{i}\left(t+\delta t_{e}\right)\right\|,
\end{aligned}
$$

where II || means a vector norm. In this paper, abbreviations written in capital italic letters denote vector quantities and the variables having a dimension of length are
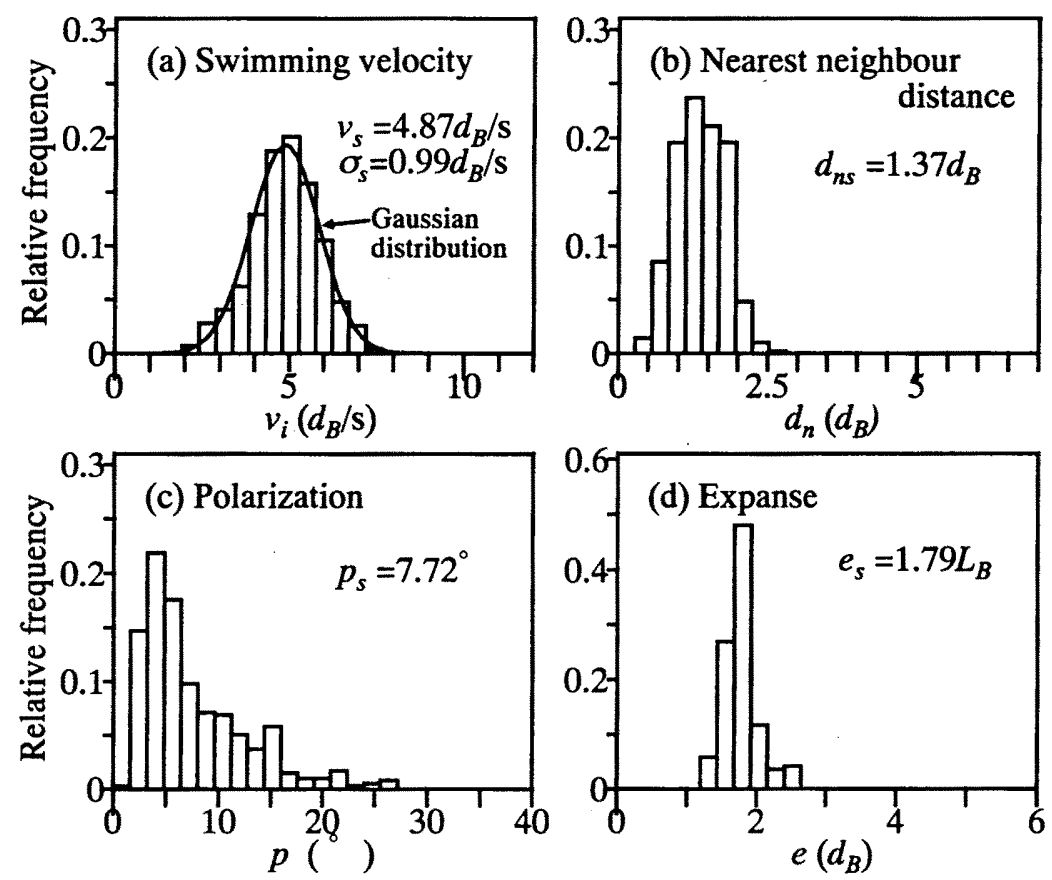

Fig. 3. Frequency distributions of the swimming velocity $v_{i}$, the nearest neighbor distance $d_{n}$, the polarization $p$, the expanse $e$ calculated from the water tank experiment of Run 1. 

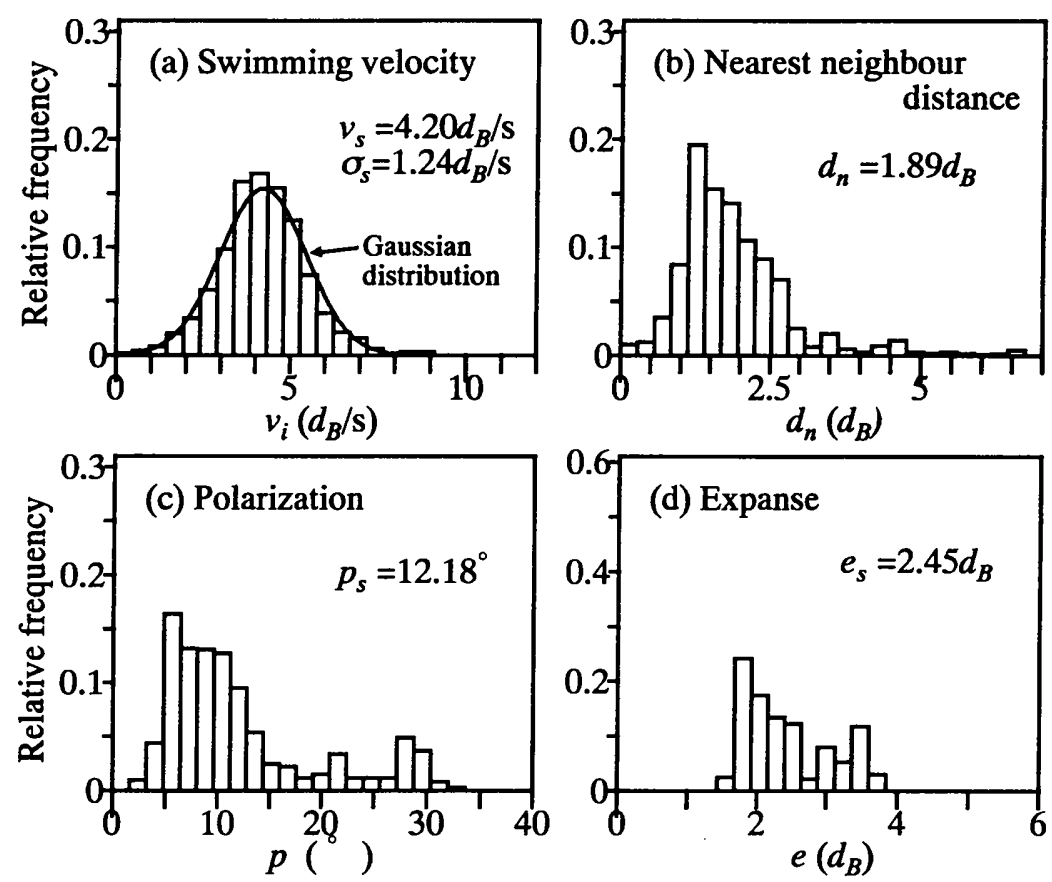

Fig. 4. Frequency distributions of the swimming velocity $v_{t}$, the nearest neighbor distance $d_{n}$, the polarization $p$, the expanse $e$ calculated from the water tank experiment of Run 2 .

Table 1. Average $v_{s}$ and standard deviation $\sigma_{s}$ of the swimming velocity, and characteristic variables $d_{n s}, p_{s}, e_{s}$ and $f_{r}$ calculated from the trajectories of individual fish in the water tank experiments of Run 1 and Run 2

\begin{tabular}{ccccccc}
\hline Run & $v_{s}\left(d_{B} / \mathrm{s}\right)$ & $\sigma_{s}\left(d_{B} / \mathrm{s}\right)$ & $d_{2 s}\left(d_{B}\right)$ & $p_{s}\left({ }^{\circ}\right)$ & $e_{s}\left(d_{B}\right)$ & $f_{r}$ \\
\hline 1 & 4.87 & 0.99 & 1.37 & 7.72 & 1.79 & 1.14 \\
2 & 4.20 & 1.24 & 1.89 & 12.18 & 2.45 & 1.24 \\
\hline
\end{tabular}

normalized by the average body-length $d_{B}$. The frequency distributions of $v_{i}$ for Run 1 and Run 2 are shown in Fig. 3(a) and Fig. 4(a). The average swimming velocity $v_{s}$ and the standard deviation $\sigma_{s}$, calculated over every individual and every time step, are summarized in Table 1. Fig. 3(a) and Fig. 4(a) reveal that the frequency distribution of $v_{i}$ is almost a Gaussian distribution $\mathrm{N}\left(v_{s}, \sigma_{s}\right)$, while Aoki (1980) studied the schooling behavior of Japanese Tamoroko (Gnathopogon elongatus) and Jack Mackerel (Trachurus japonicus) and reported that the frequency distribution of swimming velocity could be described as a Gamma distribution. The cruising velocities measured for other fish species in the previous studies ranged from 2 to 4 body-lengths per second. The average swimming velocity $v_{s}$, shown in Table 1 , which roughly corresponds to cruising velocity, is slightly over the upper limit of the previous studies. The differences 
from the previous studies may be attributed to the differences of fish species and experimental conditions.

\section{Nearest neighbor distance}

Several variables were introduced to quantify the global structure and the typical characteristics of a fish school in our experiments: the nearest neighbor distance $d_{n}$, the polarization $p$, the expanse $e$ and the fractal dimension $f_{r}$. In this paper, these variables are called the characteristic variables and will be used to evaluate the fitness in the calculation of a SGA that will be introduced to the optimization of the model structure.

The nearest neighbor distance $d_{n}$ characterizes not only the typical distance between individuals but also the cohesiveness of a fish school. It is defined as the arithmetic mean of the distances of every fish to its nearest neighbor (Huth and Wissel, 1994). The frequency distributions of $d_{n}$ for Run 1 and Run 2 are shown in Fig. 3(b) and Fig. 4(b). The average nearest neighbor distance $d_{n s}$, calculated over every time step, is summarized in Table 1 . The past study revealed that $d_{n}$ varied among the species and that the typical values ranged from 0.3 to 3 body-lengths (Huth and Wissel, 1992). The values of $d_{n}$ in both Run 1 and Run 2 are almost within this range and the values of $d_{r s}$ indicate that the school is more cohesive for Run 1 than for Run 2.

\section{Polarization}

The polarization $p$ quantifies the intensity of the parallel orientation of a fish school and is defined as the arithmetic mean of the angle deviations of every fish to the average swimming direction of the fish school (Huth and Wissel, 1992):

$$
\begin{aligned}
& p(t)=\frac{1}{n} \sum_{i=1}^{n} \angle\left(V_{i}^{0}(t), V_{s}(t)\right), \\
& V_{s}(t)=\sum_{i=1}^{n} V_{i}^{0}(t),
\end{aligned}
$$

where $\angle$ denotes an interior angle between two vectors and $V_{\imath}^{t}(t)$ is a unit velocity vector (an orientation vector) of individual $i$ at time step $t$. The average swimming direction $V_{s}(t)$ of the school is calculated by adding the orientation vector $V_{i}^{\circ}(\mathrm{t})$ of each fish as defined by equation (4). The polarization $p$ has values between $0^{\circ}$ and $90^{\circ}$. The values of $p=0^{\circ}$ and $p=90^{\circ}$ correspond to the optimally parallel (polarized) and the maximally confused schools, respectively. The frequency distributions of $p$ for Run 1 and Run 2 are depicted in Fig. 3(c) and Fig. 4(c). The average polarization $p_{s}$, calculated over every time step, is summarized in Table 1. It was reported that fish schools possessing a high parallel orientation had the values of $p$ between $10^{\circ}$ and $20^{\circ}$ (Huth and Wissel, 1992). The polarizations $p$ of both Run 1 and Run 2 are almost within this range and the values of $p_{s}$ reveal that the school is more polarized for Run 1 than for Run 2 as presumed by observing Figs. 1 and 2.

\section{Expanse}

The expanse $e$ is defined as the root mean square of the distances of every fish to the center of the mass of a fish school (Huth and Wissel, 1992): 


$$
\begin{gathered}
e(t)=\left[\frac{1}{n} \sum_{i=1}^{n}\left\|X_{i}(t)-X_{s}(t)\right\|^{2}\right]^{1 / 2}, \\
X_{s}(t)=\frac{1}{n} \sum_{i=1}^{n} X_{i}(t),
\end{gathered}
$$

where $X_{s}(t)$ is the center of the mass of the fish school. The frequency distributions of $e$ for Run 1 and Run 2 are shown in Fig. 3(d) and Fig. 4(d). The average expanse $e_{s}$, calculated over every time step, is summarized in Table 1 . The numerical simulations carried out by Huth and Wissel (1992) revealed that the typical expanse $e$ had the values
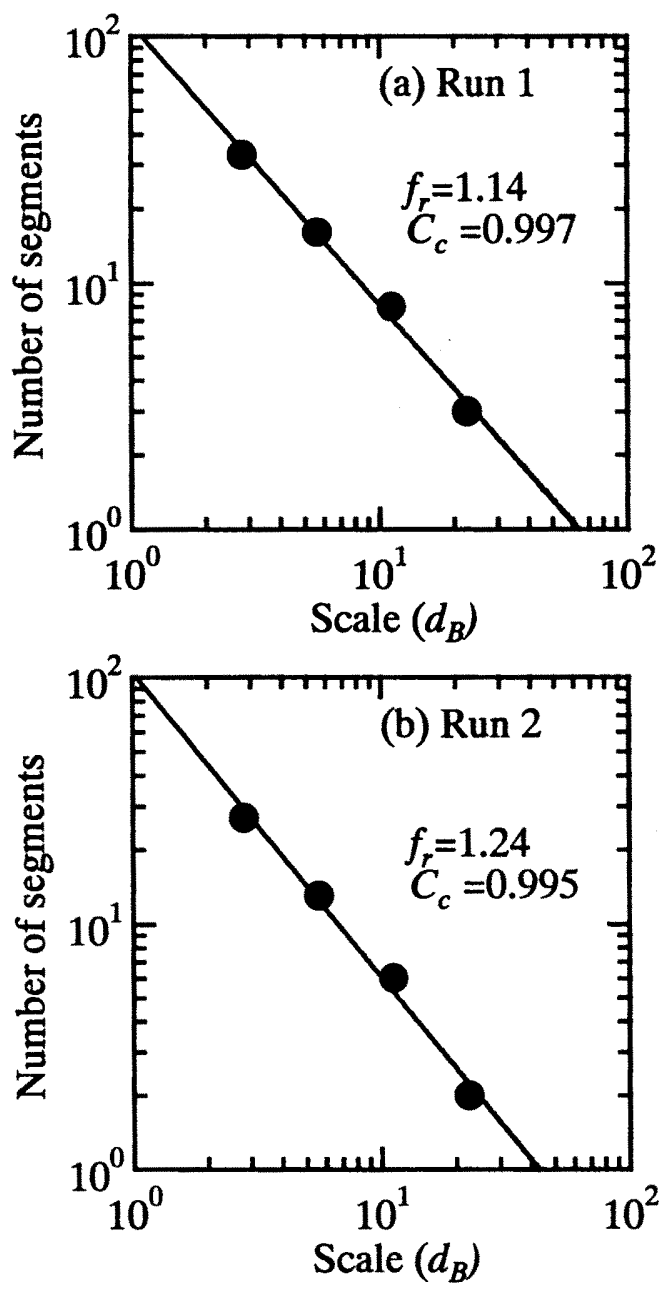

Fig. 5. Fractal dimensions $f_{r}$ calculated for the trajectories of the center of the mass of the fish school in the water tank experiments of Run 1 and Run $2 ; C_{c}$ denotes a correlation coefficient. 
of about 1 body-length when the fish school stayed together. They also indicated that the expanse $e$ increased to a value of 4 body-lengths as the fish school dispersed. According to their results, the fish schools of Run 1 and Run 2 are slightly dispersed.

\section{Fractal dimension}

The fractal dimension was originally proposed to quantify the self-similarity of figures, structures and phenomena having no characteristic length which are generally called fractal (Takayasu, 1986). On the other hand, in this paper, the fractal dimension $f_{r}$ was introduced to characterize the straightness of trajectory of the center of the mass of a fish school. The fractal dimension $f_{r}$ is shown in Fig. 5 and Table 1. As directly surmised by Figs. 1 and 2 , the values of $f_{r}$ indicate that the straightness of trajectory of fish school is stronger for Run 1 than for Run 2.

\section{Mathematical modeling}

The possibility of the behavioral pattern model based on Huth and Wissel (1992) to the reproduction of the schooling behavior observed in the water tank experiments will be examined in the following section.

Based on biological facts, four fundamental assumptions were provided in the behavioral pattern model.

(1) Every fish swims according to the same behavioral model, which guarantees that the model fish school moves without a leader.

(2) The motion of each fish is influenced only by its neighbors, which takes into account that vision and lateral line are considered to be the most important senses for schooling.

(3) The motion of the model fish school is not affected by external influence, which means that the school has no destination and no obstacle.

(4) Random influences are taken into account for each individual fish. The position and the velocity of each fish are thereupon stochastically determined.

In the behavioral pattern model, the new position $X_{i}\left(t+\delta t_{n}\right)$ and the new orientation $V_{i}^{0}\left(t+\delta t_{n}\right)$ of an individual $i(=1 \cdots, n)$ on a two-dimensional plane are calculated using the position $X_{i}(t)$ and the orientation $V_{i}^{0}(t)$ at the preceding time step:

$$
\begin{gathered}
X_{i}\left(t+\delta t_{n}\right)=X_{i}(t)+\delta t_{n} \cdot V_{i}\left(t+\delta t_{n}\right), \\
V_{i}\left(t+\delta t_{n}\right)=V_{i}^{0}\left(t+\delta t_{n}\right) \cdot v_{i}\left(t+\delta t_{n}\right), \\
V_{i}^{0}\left(t+\delta t_{n}\right)=\left(\begin{array}{ll}
\cos \alpha_{i}(t) & -\sin \alpha_{i}(t) \\
\sin \alpha_{i}(t) & \cos \alpha_{i}(t)
\end{array}\right) V_{i}^{0}(t),
\end{gathered}
$$

where $\delta t_{n}$ is an interval of a time step, $V_{i}(t), v_{i}(t)$ and $\alpha_{i}(t)$ are a velocity vector, a scalar velocity (a norm of $V_{i}(t)$ ) and a turning angle of individual $i$ at time step $t$, respectively.

In our numerical simulations, the value of $1 / 3 \mathrm{~s}$ was adopted for $\delta t_{n}$ by referring to the experimental data of Partridge (1980, 1981) and Partridge and Pitcher (1980) concerning the reaction time of fish. In accordance to the fundamental assumption that the motion of the model fish school is not affected by external influence, the fish school was simulated as a freely swimming school in the boundless area with no obstacles in spite of the presence of side walls in the water tank experiments. The velocity $v_{i}(t)$ was given by the 

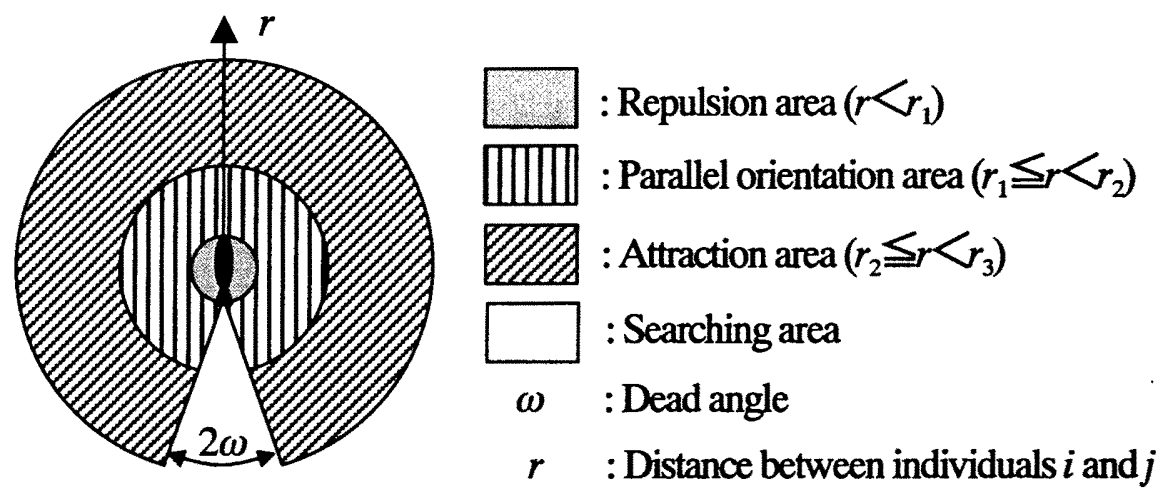

Fig. 6. Definition of four ranges of the basic behavioral pattern around individual $i$. Black fish denotes individual $i$.

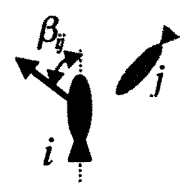

(a) Repulsion

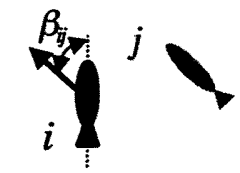

(b) Parallel orientation

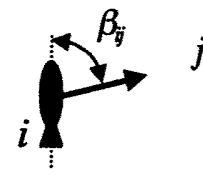

(c) Attraction

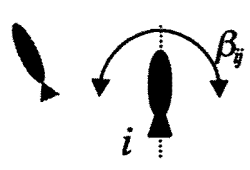

(d) Searching

Fig. 7. Schematic diagram of the initial and the new swimming directions of individual $i$ (black-colored) determined by the range where neighbor $j$ (gray-colored) positions.

random numbers with the typical distribution obtained from the water tank experiments, i.e. a Gaussian distribution $\mathrm{N}\left(v_{s}, \sigma_{s}\right)$ as depicted in Figs. 3(a) and 4(a), which means that $v_{i}(t)$ was chosen independently of other fish and other time steps. The turning angle $\alpha_{i}(t)$ was determined by the basic behavioral patterns as described as follows.

\section{Influence of neighbors}

The basic behavioral patterns determine how a fish would act if it had only one neighbor fish. Aoki (1982), Huth and Wissel (1992) and Reuter and Breckling (1994) divided the 2-dimensional space around an individual fish into four ranges in accordance with possible positions of its neighbor fish: the repulsion area, the parallel orientation area, the attraction area and the searching area. Fig. 6 shows the ranges of the basic behavioral pattern around individual $i$. In this figure, $r$ denotes the distance between individual $i$ and its neighbor $j$ and $\omega$ is a dead angle. The repulsion area is the range where the position of neighbor $j$ is in $r<r_{1}$ and not in the dead angle area, the parallel orientation area in $r_{1} \leq r<r_{2}$ and not in the dead angle area, the attraction area in $r_{2} \leq r$ $<r_{3}$ and not in the dead angle area, and the searching area in $r_{3} \leq r$ or in the dead angle area. Fig. 7 shows the initial and the new swimming directions of individual $i$, determined 


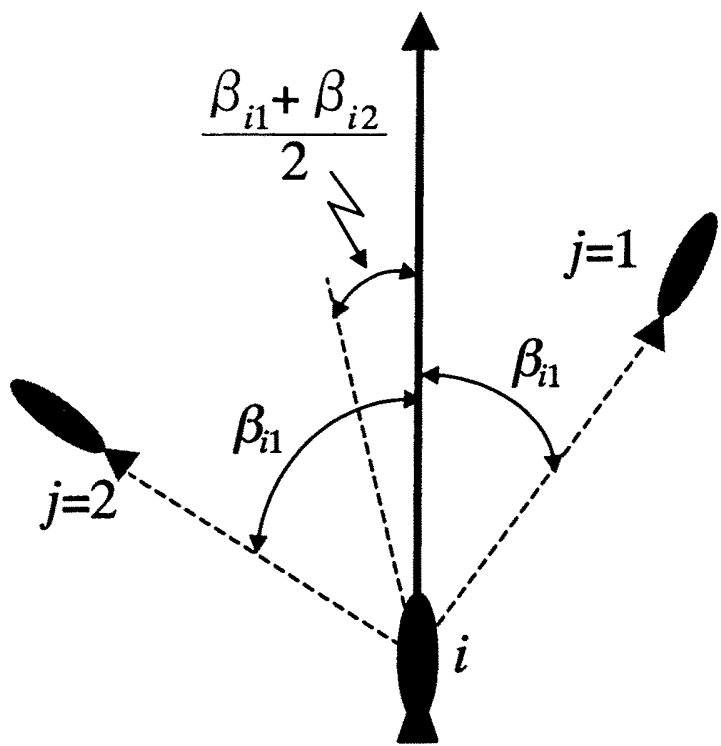

Fig. 8. Schematic of mixture of influences from two neighbors (gray-colored) around individual $i$ (black-colored) using an arithmetic mean.

by the range where neighbor $j$ positions itself. The angle $\beta_{i j}$ is an influence angle of individual $i$ due to neighbor $j$. In the repulsion area, individual $i$ swims perpendicularly away from the swimming direction of neighbor $j$. Individual $i$ swims in the same direction as neighbor $j$ in the parallel orientation area and in the direction of neighbor $j$ in the attraction area. In the searching area, individual $i$ cannot perceive its neighbor and it turns around between $-90^{\circ}$ and $90^{\circ}$ by chance. The uniform random numbers were used for the calculation of the searching behavior.

\section{Mixture of the neighbor influences}

The influence of several neighbors was mixed by using an arithmetic mean $\beta_{i j}{ }^{m}$ of the influence angles $\left\{\beta_{i j}, j=1 \cdots, n_{b}\right\}$ in which $n_{b}(\leq n-1)$ is the number of neighbors affecting the swimming behavior of individual $i$. The mixture element in the case of two neighbors is illustrated in Fig. 8 . The choosing of $n_{b}$ neighbors among $(n-1)$ individuals was decided using a certain priority rule. Four priority rules were studied in this paper: the front priority, the side priority, the distance priority and the random choosing. Individual $i$ chooses $n_{b}$ neighbors that are in a fronter position of individual $i$ in the front priority rule and that are in a closer position to the direction perpendicular to the orientation of individual $i$ in the side priority rule. The nearest neighbors are chosen from the distance priority rule and the individual $i$ randomly chooses $n_{b}$ neighbors in the random choosing rule.

Finally, the turning angle $\alpha_{i}$ was given by the random numbers with a Gaussian 
distribution $\mathrm{N}\left(\beta_{i j}^{m}, \sigma_{a}\right)$ in which $\sigma_{a}$ is a standard deviation of the turning angle $\sigma_{i}$.

\section{Estimation of model parameters and priority rule}

The average $v_{s}$ and the standard deviations $\sigma_{s}$ of the swimming velocity, the standard deviation $\sigma_{a}$ of the turning angle $\alpha_{i}$, the ranging parameters $r_{1}, r_{2}, r_{3}, \omega$, and the number $n_{b}$ of neighbors that affect the swimming behavior of an individual must be given to calculate the schooling behavior by use of the behavioral pattern model. The priority rule also must be determined.

The parameters $v_{s}, \sigma_{s}, \sigma_{a}$ and $r_{3}$ were directly determined from the results of the water tank experiments. The values of $v_{s}$ and $\sigma_{s}$ are shown in Table 1. The parameter $\sigma_{a}$ was estimated to be $10^{\circ}$ from the standard deviation of the turning angles calculated over every individual and every time step for Run 1 and Run 2 . The boundary distance $r_{3}$ between the attraction and the searching areas was presumed to be 50 body-lengths from the observation of the schooling fish. Other parameters $r_{1}, r_{2}, \omega, n_{b}$ and the priority rule were optimally searched by a SGA using the experimental data.

\section{Simple genetic algorithm}

A simple genetic algorithm (SGA) was incorporated into the modeling to help search for the optimal model parameters and the priority rule. A genetic algorithm (GA) is an optimization technique modeled after the biological processes of natural selection and

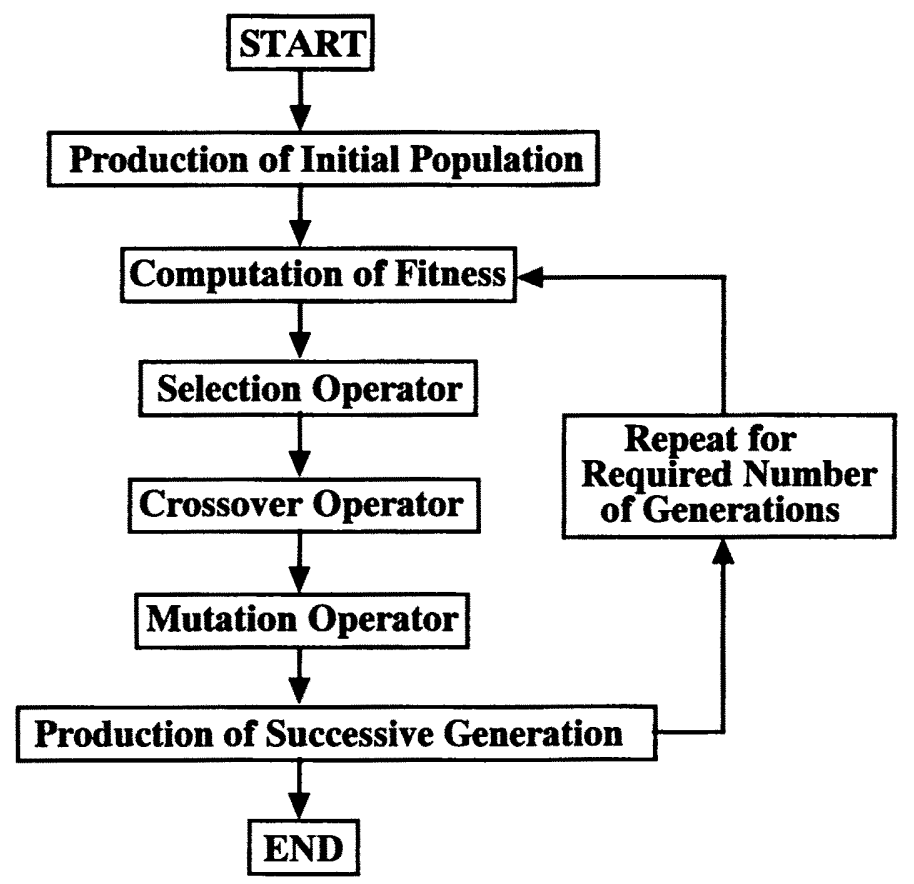

Fig. 9. Flow diagram of a simple genetic algorithm. 
evolution. The GA operates on a population of decision variable sets. Through the application of three specialized genetic operators: selection, crossover, and mutation, the GA population evolves toward an optimal solution. The basic outline of a SGA, which is a basic sort of the GAs, is shown in Fig. 9. For example, a SGA has been used by Hiramatsu et al. (1998) to determine the optimal network structure of artificial neural network model for predicting the water-stages in a tidal river. A detailed description of the genetic operations used in the SGA to search for the optimal parameters and the priority rule of the behavioral pattern model is as follows.

\section{(Step 1) Generation of an initial population}

An initial population of coded strings representing the model parameters and the priority rule is randomly generated with population size $n_{p}=60$. This coded string of 19 binary bits $\left\{b_{i}, i=1,19\right\}$ consisting of five coded substrings represents the parameters $r_{1}$, $r_{2}, \omega, n_{b}$ and the priority rule:

$$
\begin{gathered}
r_{1}=0.1 \times\left(2^{4} b_{1}+2^{3} b_{2}+2^{2} b_{3}+2 b_{4}+b_{5}\right), \\
r_{2}=r_{1}+0.5 \times\left(2^{4} b_{6}+2^{3} b_{7}+2^{2} b_{8}+2 b_{9}+b_{10}\right), \\
\omega=5 \times\left(2^{4} b_{11}+2^{3} b_{12}+2^{2} b_{13}+2 b_{14}+b_{15}\right), \\
n_{b}=2 b_{16}+2 b_{17}+1, \\
n_{p}=2 b_{18}+b_{19}+1,
\end{gathered}
$$

where $n_{p}=1,2,3$ and 4 correspond to the front priority, the side priority, the distance priority and the random choosing rules, respectively. As mentioned above, the radiuses $r_{1}$ and $r_{2}$ are normalized values by the average body-length $d_{B}$.

\section{(Step 2) Computation of the fit to the experimental data}

For each of the $n_{p}$ strings, a coded string is decoded into $r_{1}, r_{2}, \omega, n_{b}$ and the priority rule by equations (10) through (14). The fit to the experimental data is evaluated by four relative errors:

$$
e_{1}=\frac{\left|d_{n s}{ }^{n}-d_{n s}\right|}{d_{n s}}, e_{2}=\frac{\left|p_{s}{ }^{n}-p_{s}\right|}{p_{s}}, e_{3}=\frac{\left|e_{s}{ }^{n}-e_{s}\right|}{e_{s}}, e_{4}=\frac{\left|f_{r}^{n}-f_{r}\right|}{f_{r}}
$$

As mentioned in the previous section, concerning the water tank experiments, $d_{n s}, p_{s}, e_{s}$ and $f_{r}$ are the average values of the nearest neighbor distance, the polarization, the expanse and the fractal dimension. The superscript $n$ denotes the value that is obtained in the numerical simulations.

\section{(Step 3) Computation of the fitness}

The fitness $f_{t}$ of each string must be determined in order to select which strings in the population get to reproduce:

$$
f_{t}=\frac{1}{e_{1}+e_{2}+e_{3}+e_{4}} .
$$

Equation (16) means that four relative errors are equivalently treated in the calculation of fitness.

(Step 4) Generation of a new population using the selection operator 
New members of the next generation are determined by a proportionate selection method with an elite preservation strategy.

\section{(Step 5 ) The crossover operator}

One-point crossover is generally used in a SGA. Hiramatsu et al. (1998) however used uniform crossover because of its good convergence. The uniform crossover is then performed with the probability of a crossover $p_{c}=0.5$ for each pair of parent strings selected in Step 4, which means that $100 p_{c} \%$ of the pairs undergo crossover on average.

\section{(Step 6) The mutation operator}

Mutations are introduced into the population on a bit-by-bit basis at every generation to prevent the algorithm from converging to a local optimum. The mutation is performed with the probability of mutation $p_{m}=0.05$ for each bit in the strings.

\section{(Step 7) Production of successive generations}

Using Steps 2 to 6 described above, a new generation is produced. Steps 2 to 6 are repeated until the number of generations exceeds the preselected number of generations $n_{G}=60$.

\section{(Step 8) Optimal model}

The string which has the maximum fitness in the final population obtained after $n_{G}$ generations is decoded into $r_{1}, r_{2}, \omega, n_{b}$ and the priority rule by equations (10) through (14).

\section{RESULTS AND DISCUSSION}

The average and the standard deviation of the swimming velocity, and the characteristic variables were calculated for the simulated school and are summarized in Table 2. The fractal dimension is also shown in Fig. 10. These variables were obtained at $\delta t_{n}=1 / 3 \mathrm{~s}$ interval from the trajectories of individual fish calculated by the optimal model structure. The values of $d_{n s}{ }^{n}, p_{s}{ }^{n}, e_{s}{ }^{n}$ and $f_{r}^{n}$ obtained by the numerical simulations are almost equal to those of the water tank experiments shown in Table 1 and Fig. 5. Because the swimming velocity was given by the random numbers with a Gaussian distribution $\mathrm{N}\left(v_{s}, \sigma_{s}\right)$, the average value $v_{s}{ }^{n}$ and the standard deviation $\sigma_{s}{ }^{n}$ have the same values as those of the experiments. The frequency distributions of the characteristic variables obtained by the simulations are depicted in Figs. 11 and 12 . While the frequency distributions of the characteristic variables were not considered in the definition of the fitness in the optimization process with the SGA, the distributions are almost same as those of the experiments shown in Figs. 3 and 4.

Table 3 shows the optimal model parameters and the priority rule estimated by the

Table 2. Average $v_{s}^{n}$ and standard deviation $\sigma_{s}^{n}$ of the swimming velocity, and characteristic variables $d_{n s}{ }^{n}, p_{s}{ }^{n}, e_{s}{ }^{n}$ and $f_{r}^{n}$ calculated from the trajectories of individual fish in the numerical simulations of Run 1 and Run 2

\begin{tabular}{ccccccc}
\hline Run & $v_{s}{ }^{n}\left(d_{B} / \mathrm{s}\right)$ & $\sigma_{s}{ }^{n}\left(d_{B} / \mathrm{s}\right)$ & $d_{n s}{ }^{n}\left(d_{B}\right)$ & $p_{s}{ }^{n}\left(^{\circ}\right)$ & $e_{s}{ }^{n}\left(d_{B}\right)$ & $f_{r}{ }^{n}$ \\
\hline 1 & 4.88 & 0.98 & 1.38 & 9.39 & 1.73 & 1.06 \\
2 & 4.22 & 1.24 & 1.84 & 12.12 & 2.36 & 1.26 \\
\hline
\end{tabular}



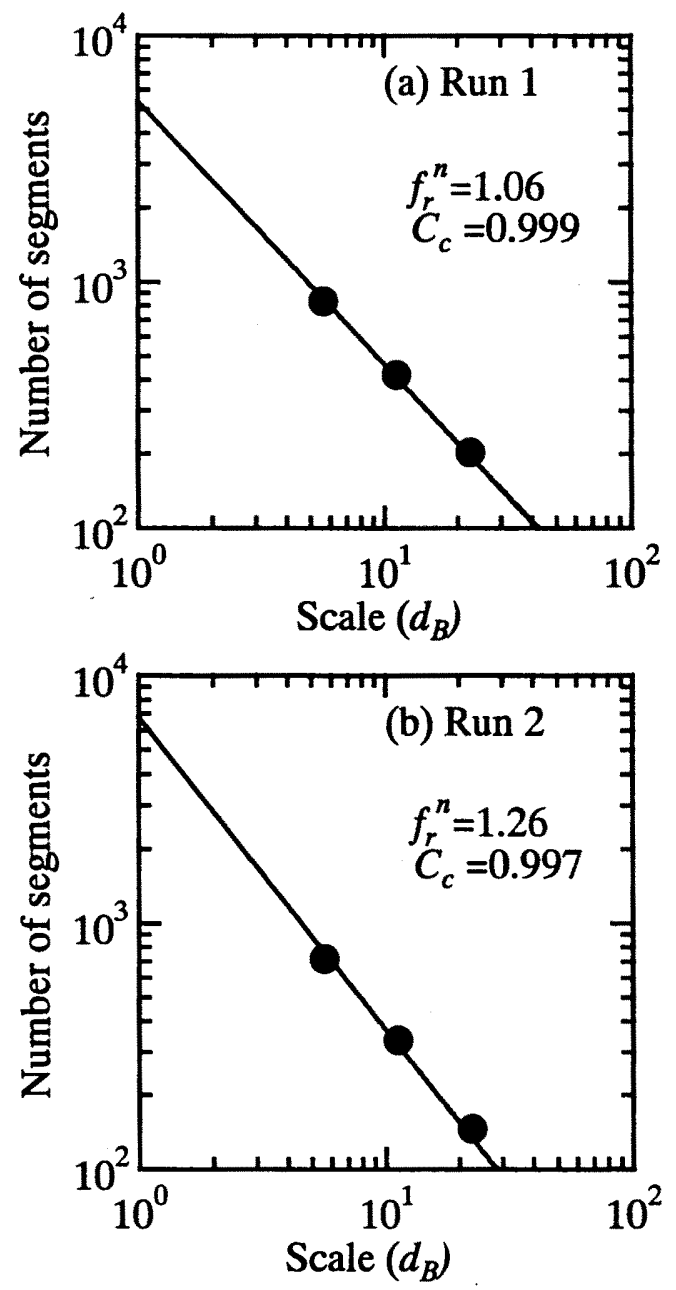

Fig. 10. Fractal dimensions $f_{r}$ calculated for the trajectories of the center of the mass of the simulated fish school in Run 1 and Run $2 ; C_{c}$ denotes a correlation coefficient.

SGA process. The radiuses $r_{1}$ and $r_{2}$ were larger for Run 1 than for Run 2 as presumed by Figs. 1 and 2. Aoki (1982), Huth and Wissel (1992) and Reuter and Breckling (1994) used the dead angle $\omega$ of $30^{\circ}$ in their numerical simulations with the behavioral pattern model, but the value of $\omega$ is found from optimization to be $0^{\circ}$. The front priority rule and the value of $n_{b}=4$ were adopted from the optimizations for both Run 1 and Run 2 . Because the influence of neighbors was mixed by using an arithmetic mean of the influence angles of $n_{b}$ neighbors in our model, four priority rules should have the same result in the case of $n_{b}=n-1(=4)$. The optimizations preliminarily carried out for the fish 

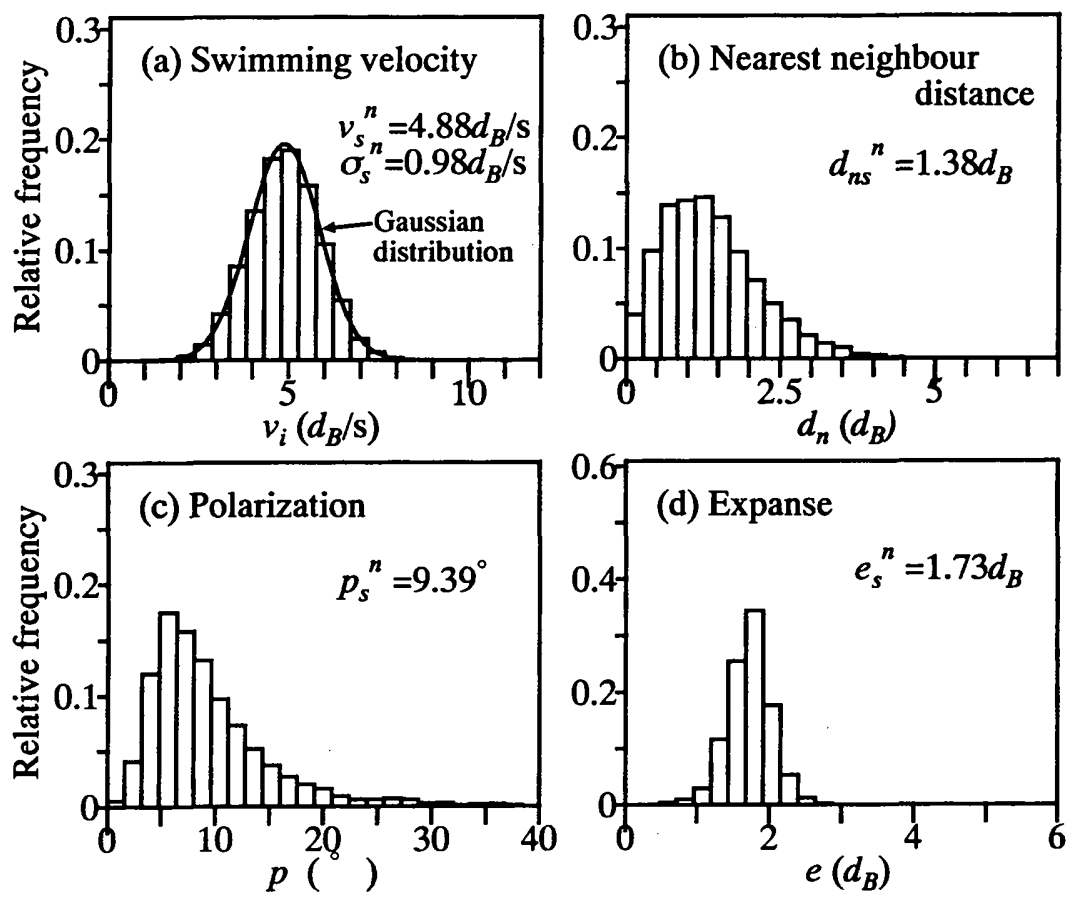

Fig. 11. Frequency distributions of the swimming velocity $v_{i}$, the nearest neighbor distance $d_{n}$, the polarization $p$, the expanse $e$ calculated from the numerical simulation of Run 1.

Table 3. Optimal model parameters and the priority rule estimated by the SGA process for Run 1 and Run 2

\begin{tabular}{cccccc}
\hline Run & $r_{1}\left(d_{B}\right)$ & $r_{2}\left(d_{B}\right)$ & $\omega\left(^{\circ}\right)$ & $n_{b}$ & Priority rule \\
\hline 1 & 0.10 & 4.60 & 0 & 4 & Front \\
2 & 0.90 & 5.90 & 0 & 4 & Front \\
\hline
\end{tabular}

school with 10 individuals $(n=10)$ yielded the maximum value of $n_{b}=9(=n-1)$ and the front priority rule.

Fig. 13 shows an example of the fish schools of Run 1 obtained by the water tank experiment and the numerical simulation. Both schools show a similar trend in schooling behavior, but the shape of the school appears to be slightly longer in the swimming direction of the school in the experiment rather than in the simulation. This is because the external force, due to the existence of side wall, was not considered in the simulations as described in the fundamental assumptions and the fish school swam freely while perceiving no obstacles.

In this paper, a mathematical model was proposed to describe the behavior of fish 

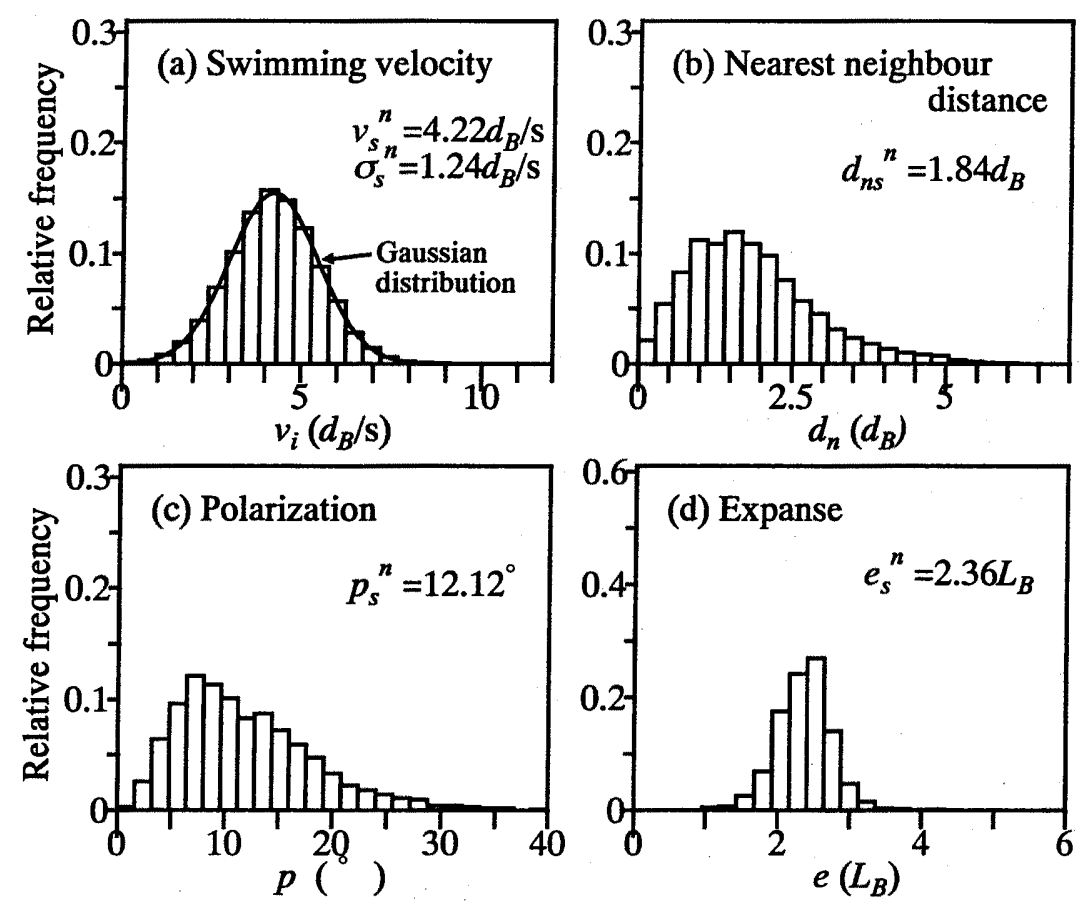

Fig. 12. Frequency distributions of the swimming velocity $v_{i}$, the nearest neighbor distance $d_{n}$, the polarization $p$, the expanse $e$ calculated from the numerical simulation of Run 2 .

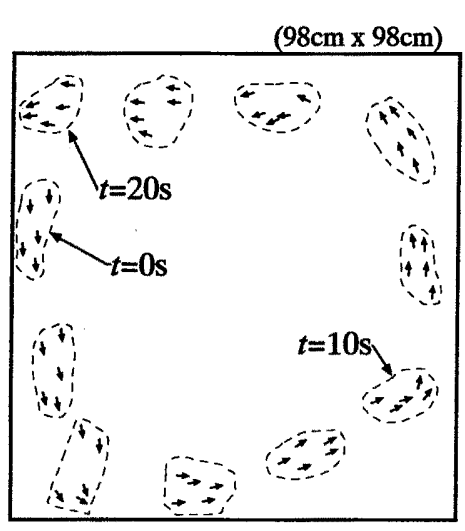

(a) Observed fish school in a water tank.<smiles>[Ge]=c1[c+]ccc1</smiles><smiles>C1=CC=[As]=1</smiles>

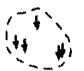<smiles></smiles><smiles>C1=[As]C[Te]1</smiles>

(b) Simulated fish school.

Fig. 13. An example of the fish schools obtained by the water tank experiment and the numerical simulation of Run 1. 
schooling in a water tank without the presence of flow. First, the water tank experiments were executed and the video images of schooling Japanese Medaka in the water tank were analyzed to investigate the interactions between individuals. The behavioral pattern model was then applied to simulate the schooling phenomenon and the SGA was incorporated into the modeling to help search for the optimal parameters and the priority rule. The results of the numerical simulations showed that the real behavior of the schooling fish of Japanese Medaka was well reproduced by the behavioral pattern model incorporating the SGA with the fitness defined by the characteristic variables $d_{n s}, p_{s}, e_{s}$ and $f_{r}$. It was also suggested that the model could be furthermore optimized in order to reproduce the real fish school more accurately if other characteristic variables were introduced into the fitness in the SGA process.

In the next step of our study, the behavioral pattern model should be improved by considering external influences such as flow and obstacles, and other variables that represent the characteristics of the real fish school, should be searched in order to improve the model optimization by using the SGA.

\section{ACKNOWLEDGEMENT}

We wish to thank Dr. Yuji Oshima of our faculty for his precious comments and help in conducting the water tank experiment using Japanese Medaka. Partial financial support for this study was provided by a Grant-in-Aid for Scientific Research from the fund of Japan Society for the Promotion of Science (no. 11660245 and no. 12460107).

\section{REFERENCES}

Aoki, I. 1980 An analysis of the schooling behavior of fish: internal organization and communication process. Bull. Ocean Res. Inst. Univ. Tokyo, 12: 1-65

Aoki, I. 1982 A simulation study on the schooling mechanism in fish. Bull. Jap. Soc. Sci. Fish., 48: 1081-1088

Hiramatsu, K., S. Shikasho and K. Mori 1998 The use of genetic algorithm and artificial neural network for the short-term prediction of water-Stages in a tidal river. Proceedings of the 11 th Congress of Asia and Pacific Division of the International Association for Hydraulic Research: 587-595

Huth, A. and C. Wissel 1992 The Simulation of the movement of fish schools. J. Theor. Biol, 156: 365-385

Huth, A. and C. Wissel 1994 The simulation of fish schools in comparison with experimental data. Ecol. Model., 75/76: 135-145

Niwa, H. 1994 Self-organizing dynamic model of fish schooling. J. Theor. Biol., 171: 123-136

Okubo, A. 1986 Dynamical aspects of animal grouping, swarms, schools, flocks and herds. Adv. Biophys., 22: 1-94

Partridge, B. L. 1980 The effect of school size on the structure and dynamics of minnow schools. Anim. Behav., 28: $68-77$

Partridge, B. L. and T. J. Pitcher 1980 The sensory basis of fish schools: relative roles of lateral line and vision. J. Comp. Physiol. A, 135: 315-325

Partridge, B. L. 1981 Internal dynamics and the interrelations of fish in schools. J. Comp. Physiol. A, 144: $313-325$

Reuter, H. and B. Breckling 1994 Selforganization of fish schools: an object-oriented model. Ecol. Model., 75/76: $135-145$

Sannomiya, N. and K. Matuda 1984 A mathematical model of fish behavior in a water tank. IEEE T. Syst. Man Cyb., SMC-14: 157-162

Takayasu, H. 1986 Fractal. Asakura Shoten, Tokyo (Japan) (in Japanese) 